\title{
Impacto da exposição à sífilis materna no sistema auditivo de recém-nascidos
}

\section{Impact of exposure to maternal syphilis on the newborn's auditory}

\section{system}

\author{
Georgea Espindola Ribeiro' (1D, Daniela Polo Camargo da Silva² (D), Jair Cortez Montovani ${ }^{1}$ (D), \\ Regina Helena Garcia Martins ${ }^{1}$ (1)
}

\begin{abstract}
RESUMO
Objetivo: Avaliar os achados audiológicos de recém-nascidos expostos à sífilis materna, tratada adequadamente na gestação. Métodos: Estudo de coorte única não concorrente, realizado em um hospital público. Fizeram parte da amostra recém-nascidos filhos de mães que apresentaram sorologia positiva para sífilis e que realizaram o tratamento adequado durante a gestação, compondo o grupo estudo, e recém-nascidos sem indicadores de risco para deficiência auditiva, grupo comparação. Resultados: Participaram do estudo 90 recém-nascidos, sendo 41 do grupo estudo e 49 do grupo comparação. No exame de emissões otoacústicas por estímulo transiente, todos apresentaram presença de resposta bilateralmente e, na comparação das amplitudes de respostas nas bandas de frequências de 3,0 $\mathrm{kHz}, 3,5 \mathrm{kHz}$ e 4,0 kHz observou-se menor amplitude de resposta no grupo exposto, com diferença estatisticamente significativa para a frequência de $4 \mathrm{kHz}$ à direita. $\mathrm{Na}$ análise das respostas do exame de potencial evocado auditivo de tronco encefálico, não se observou assimetria de resposta entre as orelhas que sugerisse alteração retrococlear e nem diferenças nos valores das latências absolutas ou intervalos interpicos, entre os grupos. Conclusão: As respostas eletroacústicas foram discretamente inferiores nos recém-nascidos exposto à sífilis materna, enquanto que as respostas eletrofisiológicas foram semelhantes às encontradas na população de baixo risco para deficiência auditiva.
\end{abstract}

Palavras-chave: Sífilis congênita; Sífilis; Audição; Eletrofisiologia; Lactente

\begin{abstract}
Purpose: To evaluate the audiological findings of newborns exposed to maternal syphilis, properly treated during pregnancy. Methods: This was a single, non-concurrent cohort study conducted in a public hospital. The sample included newborns whose mothers had positive syphilis serology and who underwent adequate treatment during pregnancy, composing the study group, and newborns without risk indicators for hearing loss, as a comparison group. Results: Ninety newborns participated in the study, 41 were the study group and 49 were the comparison group. In the transient evoked otoacoustic emissions test, all newborns showed a bilateral response, and when comparing the amplitudes of responses in the $3.0 \mathrm{kHz}, 3.5 \mathrm{kHz}$ and $4.0 \mathrm{kHz}$ frequency bands, a smaller amplitude of response in the studied group with a statistically significant difference for the frequency of $4 \mathrm{kHz}$ on the right was observed. An analysis of the response of the brainstem auditory evoked potential test showed no asymmetry of response between the ears that suggest a retrocochlear alteration, nor difference in the values of absolute latencies or interpeak intervals between groups. Conclusion: The electroacoustic responses were slightly lower in newborns exposed to maternal syphilis, whereas their electrophysiological responses were similar to those found in the population at low risk for hearing loss.
\end{abstract}

Keywords: Syphilis, Congenital; Syphilis; Hearing; Electrophysiology; Infant

\footnotetext{
Trabalho realizado na Faculdade de Medicina de Botucatu, Universidade Estadual Paulista "Júlio de Mesquita Filho" - UNESP - Botucatu (SP), Brasil.

${ }^{1}$ Departamento de Oftalmologia, Otorrinolaringologia e Cirurgia de Cabeça e Pescoço, Faculdade de Medicina de Botucatu, Universidade Estadual Paulista "Júlio de Mesquita Filho" - UNESP - Botucatu (SP), Brasil.

${ }^{2}$ Departamento de Fonoaudiologia, Universidade Federal de Santa Catarina - UFSC - Florianópolis (SC), Brasil.

Conflito de interesses: Não.

Contribuição dos autores: GER foi responsável pela concepção e delineamento do estudo, análise e interpretação dos dados e elaboração da redação; DPCS foi responsável pela análise e interpretação dos dados, revisão do artigo de forma intelectualmente importante e aprovação final da versão a ser publicada; JCM foi responsável pela análise e interpretação dos dados, revisão do artigo de forma intelectualmente importante e aprovação final da versão a ser publicada; RHGM foi responsável pela orientação, revisão e aprovação da versão final a ser publicada.
}

Financiamento: Nada a declarar.

Autor correspondente: Georgea Espindola Ribeiro. E-mail: georgea espindola@hotmail.com

Recebido: Março 28, 2021; Aceito: Junho 28, 2021 


\section{INTRODUÇÃO}

Os primeiros anos de vida são primordiais para a aquisição e desenvolvimento das habilidades auditivas e de linguagem, pois nessa fase ocorre o processo maturacional do sistema auditivo e da plasticidade neuronal da via auditiva. Dessa forma, torna-se importante o diagnóstico precoce de qualquer alteração audiológica para minimizar seus impactos negativos na vida do indivíduo ${ }^{(1)}$.

A exposição à sífilis no período neonatal é considerada um indicador de risco para perda auditiva por afetar a orelha interna, ocasionando periostite, atrofia do órgão de Corti e hidropsia endolinfática do labirinto membranoso ${ }^{(1-3)}$. Tais alterações podem afetar principalmente, o gânglio espiral e fibras nervosas do oitavo par, acarretando perda auditiva do tipo sensorioneural, de caráter progressivo e de manifestação tardia ${ }^{(2,3)}$.

A sífilis congênita foi praticamente erradicada após a era da penicilina. Porém, nos últimos dez anos, houve um progressivo aumento mundial da sua incidência ${ }^{(4,5)}$. Em 2018, no Brasil, a taxa de detecção de sífilis em gestantes foi de 21,4/1.000 nascidos vivos e a incidência de sífilis congênita foi de $9,0 / 1.000$ nascidos vivos ${ }^{(6)}$.

Vale ressaltar que essa discrepância entre o número de gestantes com sífilis e de recém-nascidos com sífilis congênita se deve ao fato de que, apesar da gestante estar infectada, o feto não necessariamente estará contaminado. Isso dependerá da ocorrência, ou não, da disseminação hematogênica do Treponema pallidum para o concepto por via transplacentária (transmissão vertical), ocasionando, de fato, a sífilis congênita ${ }^{(4-7)}$.

O aumento dos casos de sífilis, de forma global, é atribuído, em sua maioria, à ausência do uso de preservativos, uso indiscriminado de drogas e diversidade de parceiros sexuais. Nessa mesma perspectiva, observa-se, também, uma elevação nas taxas de detecção prévia de sífilis em gestantes, indicando a adoção de critérios mais sensíveis e eficazes para identificação precoce de sífilis materna, ainda no pré-natal, o que possibilita o tratamento precoce, evitando-se, ao máximo, a contaminação transplacentária ao feto pelo Treponema pallidum ${ }^{(8,9)}$.

A sífilis, quando não tratada, ou inadequadamente tratada durante a gestação, pode levar à sífilis congênita. Entretanto, os recém-nascidos podem não apresentar sintomas e alguns recém-nascidos infectados nunca desenvolvem quaisquer problemas durante toda a sua vida ${ }^{(10)}$. No caso de recém-nascidos sintomáticos, manifestações de sífilis congênita são classificadas como de início precoce ou $\operatorname{tardio}^{(3,10)}$.

A sífilis congênita precoce surge até o segundo ano de vida, ainda que mais da metade dessas crianças sejam assintomáticas ao nascimento ${ }^{(6,11)}$. Por outro lado, o recém-nascido pode apresentar várias intercorrências, como prematuridade, baixo peso, icterícia, anemia e/ou deficit auditivo ${ }^{(11)}$.

A sífilis congênita tardia, por sua vez, surge após o segundo ano de vida, com manifestações clínicas raras, geralmente associadas à tríade de Hutchinson (alteração na coroa dentária, conhecida como "dentes de Hutchinson", ceratite intersticial e lesão do VIII par craniano) $)^{(3)}$. No entanto, com o advento dos antibióticos, a perda de audição tem sido o único sintoma ${ }^{(2)}$.

Sendo assim, toda gestante diagnosticada com sífilis deve iniciar o tratamento, a fim de evitar a sífilis congênita, fazendo uso da penicilina benzatina em doses diferenciadas, conforme a fase da infecção materna ${ }^{(6,11,12)}$. Segundo dados do Ministério da Saúde ${ }^{(6)}$, o tratamento adequado de sífilis na gestação é realizado com a medicação recomendada, havendo declínio dos títulos ao longo do seguimento, o que é observado por meio de testes não treponêmicos. Ademais, a intervenção deve ser realizada até 30 dias antes do parto ${ }^{(6,11,12)}$.

Ainda que ocorra tratamento efetivo contra a sífilis materna, os recém-nascidos expostos à sífilis são submetidos a exames físicos e a testes sorológicos, logo ao nascimento ${ }^{(6,12)}$. Mesmo não havendo indícios de transmissão, os recém-nascidos assintomáticos cujas mães tenham feito a intervenção adequada ao longo da gestação devem ser acompanhados até que o anticorpo treponêmico, adquirido passivamente, desapareça, o que só ocorre por volta dos 18 meses de vida ${ }^{(6,11,12)}$.

Do ponto de vista audiológico, todos os recém-nascidos expostos à sífilis materna, independentemente de ter, ou não, havido tratamento adequado, devem realizar avaliação e monitoramento audiológico semestral até os dois anos de idade, bem como a execução de testes treponêmicos após os 18 meses $^{(6,11,12)}$. Porém, ainda é necessário conhecer o real comprometimento auditivo que a exposição à sífilis materna pode trazer, principalmente quando há o tratamento adequado da doença ainda na gestação ${ }^{(13,14)}$.

Assim, o objetivo deste estudo foi avaliar os achados audiológicos de recém-nascidos expostos à sífilis materna.

\section{MÉTODO}

Este trabalho foi aprovado pelo Comitê de Ética em Pesquisa da Faculdade de Medicina de Botucatu da Universidade Estadual Paulista "Júlio de Mesquita Filho" - UNESP (processo ${ }^{\circ}$ 1.759.185). Os pais ou responsáveis legais que concordaram em participar do estudo assinaram o Termo de Consentimento Livre e Esclarecido.

Este foi um estudo de coorte não concorrente, realizado em um hospital público, de janeiro de 2017 a março de 2019.

\section{Sujeitos}

Fizeram parte da amostra recém-nascidos expostos à sífilis materna, compondo o grupo estudo e recém-nascidos não expostos à sífilis como grupo comparação. Ambos os grupos foram semelhantes em relação à idade gestacional, peso ao nascimento e idade no momento da avaliação auditiva, realizada após a alta hospitalar.

Os participantes foram classificados da seguinte forma:

- Grupo exposto à sífilis materna: recém-nascidos filhos de mães que foram diagnosticadas com sífilis ainda no período gestacional. Elas foram submetidas ao tratamento completo com penicilina benzatina, conforme o estágio da doença. Foi observado declínio dos títulos nos testes não treponêmicos e o tratamento foi finalizado, no máximo, 30 dias antes do parto ${ }^{(6,11,12)}$. Os recém-nascidos apresentaram teste não treponêmico (Veneral Disease Research Laboratory - VDRL) reagente, com titulação inferior ao da mãe e VDRL líquor não reagente;

- Grupo comparação: recém-nascidos sem indicadores de risco para deficiência auditiva, com VDRL não reagente, cujas mães realizaram pré-natal adequado, sem relatos de sífilis na gestação. 
Critérios de inclusão para ambos os grupos: ter nascido no local do estudo e ter realizado, na mesma sessão, os exames de emissões otoacústicas evocadas por estímulo transiente (EOAT) e potencial evocado auditivo de tronco encefálico (PEATE) com estímulo clique (protocolo neurodiagnóstico).

Critério de exclusão para ambos os grupos: prematuridade, mãe usuária de drogas na gestação e presença de qualquer outro indicador de risco para perda auditiva, segundo o Joint Committee on Infant Hearing (2019)(1).

\section{Sequência das avaliações}

\section{Especificações técnicas das emissões otoacústicas}

Para o exame de EOAT, foram registradas as respostas de ambas as orelhas, permanecendo o recém-nascido em estado de sono natural, em sala silenciosa. Caso o recém-nascido acordasse durante o exame, o (a) responsável era orientado (a) a fazê-lo dormir novamente. O equipamento utilizado em todas as avaliações foi o OtoRead/Interacoustics, cuja sonda, com microfone acoplado, era introduzida ao meato acústico externo. Como critério de análise, o parâmetro de "passa" foi estabelecido por um protocolo padronizado, utilizando-se o estímulo clique, intensidade de $83 \mathrm{dBNPS}$, sendo avaliadas seis bandas de frequências (entre 1,5 kHz e 4,0 kHz). Para que o equipamento considerasse automaticamente o exame como "passa", foi necessário atingir os seguintes critérios: amplitude de resposta de $6 \mathrm{~dB}$, ou mais, em relação à amplitude do ruído, $\mathrm{em}$, pelo menos, três bandas de frequências.

Assim, após a impressão dos registros, foram avaliadas as amplitudes de resposta das frequências de $3,0 \mathrm{kHz}, 3,5 \mathrm{kHz}$ e $4,0 \mathrm{kHz}$, por serem as mais frequentemente observadas. Além disso, o critério de "passa" adotado no serviço prioriza a presença de resposta em três bandas de frequências consecutivas, inclusive a de $4,0 \mathrm{kHz}^{(15)}$.

\section{Especificações técnicas do PEATE-clique neurodiagnóstico}

O PEATE-clique foi realizado com o equipamento ICS Charter (GN Otometrics/Dinamarca). Após a limpeza da pele com substância abrasiva (Nuprep ${ }^{\circledR}$ ), os eletrodos de superfície da marca Neuroline ${ }^{\circledR}$ foram fixados em pontos específicos. $\mathrm{O}$ eletrodo ativo foi fixado na fronte $(\mathrm{Fz})$, os de referência, nas regiões da mastoide (M1 e M2) e o eletrodo terra foi colocado na fronte. $\mathrm{O}$ estímulo foi apresentado por meio do fone de inserção ER 3A, com estimulação monoaural com cliques filtrados (filtro passa banda high pass de $100 \mathrm{~Hz}$ e low pass de $3000 \mathrm{~Hz}$ ), duração de $100 \mu$ s e polaridade rarefeita. Foram fornecidos 2.048 cliques com tempo de análise de $15 \mathrm{~ms}$, repetidos para confirmação da reprodutibilidade das ondas. A impedância dos eletrodos foi mantida abaixo de $3 \mathrm{kOhms}$. As taxas de repetição do estímulo foram de 21,1 cliques por segundo (c/s) para cada orelha, na intensidade de $80 \mathrm{dBnNA}$. Os principais parâmetros avaliados no PEATE-clique neurodiagnóstico foram: latências absolutas das ondas I, III e V e latências interpicos I- III, III - V e I - V de ambas as orelhas, bem como a diferença interaural.

A marcação das ondas e a análise do registro foram realizadas por um fonoaudiólogo especialista em audiologia, com experiência em avaliação eletrofisiológica infantil.

\section{Estatística}

As comparações entre recém-nascidos com e sem exposição à sífilis materna, em relação às amplitudes de respostas das EOAT e as latências do PEATE-clique, foram feitas ajustando modelos de regressão linear múltipla, considerando os efeitos do gênero, idade gestacional e peso ao nascimento. As associações foram consideradas estatisticamente significativas se $\mathrm{p}<0,05$. A análise foi feita com o software SPSS v 21.0.

\section{RESULTADOS}

Participaram do estudo 90 recém-nascidos, sendo 41 do grupo exposto à sífilis materna e 49 do grupo comparação. Todos realizaram os exames de EOAT e PEATE-clique com idades entre zero e um mês de vida. Os dados da caracterização da amostra referentes a gênero, idade gestacional e peso ao nascimento estão apresentados na Tabela 1.

No exame de EOAT, todos os recém-nascidos, de ambos os grupos, apresentaram resultado "passa", indicando presença de resposta bilateralmente. Desta forma, houve a comparação das amplitudes de resposta de ambos os grupos nas bandas de frequências de $3,0 \mathrm{kHz}, 3,5 \mathrm{kHz}$ e $4,0 \mathrm{kHz}$ e observou-se aumento da amplitude das respostas em ambas as orelhas no grupo comparação, com diferença estatisticamente significativa para a frequência de $4,0 \mathrm{kHz}$ à direita $(\mathrm{p}<0,028)$ (Tabela 2).

$\mathrm{Na}$ análise do exame de PEATE-clique, não se observou assimetria de resposta entre as orelhas de ambos os grupos que sugerisse alteração retrococlear. Além disso, a comparação dos parâmetros avaliados neste exame, entre os grupos, não mostrou diferença nos valores das latências absolutas das ondas I, III e $\mathrm{V}$ e intervalos interpicos I-III, III-V e I-V de ambas as orelhas (Tabelas 3 e 4).

Tabela 1.Caracterização da amostra quanto ao gênero, idade gestacional e peso ao nascer

\begin{tabular}{|c|c|c|c|}
\hline Grupos/Variáveis & Grupo comparação (n=49) n (\%) & Grupo sífilis ( $n=41) n(\%)$ & Total 90 \\
\hline Gênero feminino & $28(57)$ & $19(46)$ & 47 \\
\hline Gênero masculino & $21(43)$ & $22(54)$ & 43 \\
\hline Idade gestacional ${ }^{a}$ & 39 s (min. 37s; máx. 41s) & 39s (min. 37s; máx. 41s) & \\
\hline Peso ao nascer ${ }^{\text {a }}$ & 3,267 kg (min. 2,165; máx. 4585) & 3,275 kg (min. 1,800; máx. 4,265) & \\
\hline
\end{tabular}

âresumo em mediana, mínimo (min.) e máximo (máx.) dos valores de idade gestacional e peso ao nascimento, semanas (s) e quilogramas (kg)

Legenda: $\mathrm{n}=$ número de sujeitos; $\%$ = percentual 
Tabela 2. Comparação entre os grupos quanto às amplitudes das emissões otoacústicas evocadas por estímulo transiente

\begin{tabular}{cccc}
\hline \multirow{2}{*}{ Frequências } & Grupo Comparação & Grupo Sífilis & \multirow{2}{*}{ Valor de $\mathbf{p}$} \\
\cline { 2 - 3 } & $14,9 \mathrm{~dB}( \pm 6,1)$ & $13,0 \mathrm{~dB}( \pm 5,8)$ & 0,139 \\
OD $3,0 \mathrm{kHz}$ & $16,0 \mathrm{~dB}( \pm 6,0)$ & $15,3 \mathrm{~dB}( \pm 5,5)$ & 0,519 \\
OD $3,5 \mathrm{kHz}$ & $14,9 \mathrm{~dB}( \pm 6,0)$ & $12,3 \mathrm{~dB}( \pm 4,9)$ & $\mathbf{0 , 0 2 8}$ \\
OD $4,0 \mathrm{kHz}$ & $13,7 \mathrm{~dB}( \pm 6,0)$ & $13,1 \mathrm{~dB}( \pm 5,3)$ & 0,628 \\
OE $3,5 \mathrm{kHz}$ & $16,0 \mathrm{~dB}( \pm 5,8)$ & $14,1 \mathrm{~dB}( \pm 6,1)$ & 0,166 \\
OE $4,0 \mathrm{kHz}$ & $14,5 \mathrm{~dB}( \pm 5,8)$ & $13,4 \mathrm{~dB}( \pm 5,3)$ & 0,473 \\
\hline
\end{tabular}

Valor de $p$ referente à comparação entre os grupos ( $p<0,05$; modelos de regressão linear múltipla)

Legenda: $\mathrm{OD}=$ orelha direita; $\mathrm{OE}=$ orelha esquerda; $\mathrm{DP}=$ desvio padrão

Tabela 3. Comparação das latências do potencial evocado auditivo de tronco encefálico entre os grupos

\begin{tabular}{|c|c|c|c|c|}
\hline & \multirow{2}{*}{ PEATE } & Grupo Comparação & Grupo Sífilis & \multirow{2}{*}{ Valor de } \\
\hline & & Média (DP) & Média (DP) & \\
\hline Orelha & I & $1,6 \mathrm{~ms}( \pm 0,1)$ & $1,6 \mathrm{~ms}( \pm 0,1)$ & 0,264 \\
\hline \multirow[t]{5}{*}{ Direita } & III & $4,4 \mathrm{~ms}( \pm 0,2)$ & $4,4 \mathrm{~ms}( \pm 0,2)$ & 0,852 \\
\hline & v & $6,6 \mathrm{~ms}( \pm 0,3)$ & $6,7 \mathrm{~ms}( \pm 0,3)$ & 0,405 \\
\hline & I-III & $2,8 \mathrm{~ms}( \pm 0,2)$ & $2,8 \mathrm{~ms}( \pm 0,2)$ & 0,406 \\
\hline & III-V & $2,2 \mathrm{~ms}( \pm 0,2)$ & $2,3 \mathrm{~ms}( \pm 0,2)$ & 0,069 \\
\hline & I-V & $5,1 \mathrm{~ms}( \pm 0,5)$ & $5,1 \mathrm{~ms}( \pm 0,3)$ & 0,637 \\
\hline
\end{tabular}

Valor de $p$ referente à comparação entre os grupos ( $p<0,05$; modelos de regressão linear múltipla)

Legenda: PEATE = potencial evocado auditivo de tronco encefálico; DP = desvio padrão; $\mathrm{ms}$ = milissegundos; I, III e V = latência absoluta das ondas; I-III, III-V e $\mathrm{I}-\mathrm{V}=$ intervalos interpicos

Tabela 4. Comparação das latências do potencial evocado auditivo de tronco encefálico entre os grupos

\begin{tabular}{|c|c|c|c|c|}
\hline & & Grupo Comparação & Grupo Sífilis & \\
\hline & PEAIE & Média (DP) & Média (DP) & valor de $p$ \\
\hline \multirow{6}{*}{$\begin{array}{c}\text { Orelha } \\
\text { Esquerda }\end{array}$} & I & $1,6 \mathrm{~ms}( \pm 0,1)$ & $1,6 \mathrm{~ms}( \pm 0,1)$ & 0,286 \\
\hline & III & $4,4 \mathrm{~ms}( \pm 0,2)$ & $4,4 \mathrm{~ms}( \pm 0,2)$ & 0,954 \\
\hline & V & $6,6 \mathrm{~ms}( \pm 0,2)$ & $6,7 \mathrm{~ms}( \pm 0,3)$ & 0,364 \\
\hline & I-III & $2,8 \mathrm{~ms}( \pm 0,2)$ & $2,8 \mathrm{~ms}( \pm 0,2)$ & 0,562 \\
\hline & III-V & $2,2 \mathrm{~ms}( \pm 0,2)$ & $2,3 \mathrm{~ms}( \pm 0,2)$ & 0,084 \\
\hline & I-V & $5,1 \mathrm{~ms}( \pm 0,4)$ & $5,1 \mathrm{~ms}( \pm 0,3)$ & 0,946 \\
\hline
\end{tabular}

Valor de $p$ referente à comparação entre os grupos ( $p<0,05$; modelos de regressão linear múltipla)

Legenda: PEATE = potencial evocado auditivo de tronco encefálico; DP = desvio padrão; $\mathrm{ms}$ = milissegundos; I, III e V = latência absoluta das ondas; I-III, III-V e $\mathrm{I}-\mathrm{V}=$ intervalos interpicos

\section{DISCUSSÃO}

Este estudo teve como objetivo verificar os achados audiológicos em recém-nascidos expostos à sífilis materna gestacional, tendo havido o tratamento completo da doença.

Ainda que a sífilis seja tratada adequadamente na gestação, os protocolos recomendam que esses recém-nascidos sejam submetidos a exames eletroacústicos e eletrofisiológicos logo após o nascimento, além do acompanhamento em longo prazo do desenvolvimento das vias auditivas ${ }^{(6,11,12,15)}$.

Sendo assim, este estudo procurou analisar os achados dos exames de EOAT e PEATE-clique entre recém-nascidos expostos à sífilis materna e recém-nascidos sem indicadores de risco, comparando-os e verificando possíveis alterações audiológicas precoces no grupo exposto a essa condição.

Como todos os recém-nascidos apresentaram resultado "passa" (ou seja, presença de resposta bilateralmente no exame de EOAT), procurou-se analisar as amplitudes de resposta das EOAT, a fim de se obter informações adicionais sobre o funcionamento das células ciliadas externas da cóclea ${ }^{(16)}$. Como resultado, observou-se que o grupo exposto à sífilis materna apresentou, de forma geral, menor amplitude de resposta, porém com diferença estatisticamente significativa apenas para a frequência de $4,0 \mathrm{kHz}$ à direita. Isso mostra um desempenho inferior nessa população, que poderá ser confirmado em estudos com maior casuística e com delineamento de coorte.

Salienta-se, também, que investigações posteriores, utilizando as emissões otoacústicas evocadas por produto de distorção na verificação do desempenho das células ciliadas externas cocleares, trarão informações adicionais para a análise de frequências acima de $4,0 \mathrm{kHz}$ até $10 \mathrm{kHz}$, concentradas na região basal da cóclea.

Além disso, ressalta-se que, neste estudo, houve apenas a análise da amplitude das EOAT por banda de frequência. Porém, estudos futuros com análises complementares de outros parâmetros intrínsecos a esse resultado final, como por exemplo, análise do ruído e da amplitude de resposta, contribuirão para o melhor conhecimento da diferença observada. 
A análise da amplitude das EOAT foi tema de várias pesquisas, mostrando ser influenciada por outros indicadores de risco, como a hiperbilirrubinemia, uso de medicação ototóxica e asfixia perinatal ${ }^{(17-19)}$.

A verificação das amplitudes de resposta permite refinar os critérios de normalidade adotados na interpretação do resultado das EOAT. Assim, menor amplitude de resposta neste exame pode refletir um achado patológico coclear, porém, ainda não está clara a relevância clínica deste achado ${ }^{(16)}$. Consequentemente, mais investigações serão necessárias para explicar tal fenômeno encontrado nos recém-nascidos expostos à sífilis materna.

Em relação ao exame de PEATE-clique na função da investigação neurodiagnóstica, não foram encontradas diferenças nas latências das ondas I, III e V, nem nos interpicos I-III, III-V e I-V. Portanto, pode-se afirmar que, para a casuística estudada, não houve diferenças entre os grupos. Apesar da amplitude das ondas não ser um parâmetro analisado na rotina clínica, sua medida poderá contribuir com informações adicionais na comparação com o grupo controle, em pesquisas futuras. Esses dados concordam com estudo realizado por Gleich et al. ${ }^{(13)}$, que também não encontrou relação causal entre recém-nascidos com evidência sorológica para sífilis e alteração no PEATE.

Portanto, a sincronia neural das estruturas auditivas em nível de tronco não se mostrou afetada em curto prazo pela exposição à sífilis materna, quando adequadamente tratada ${ }^{(20,21)}$.

É importante destacar que, muitas vezes, o termo "sífilis congênita" é visto de forma generalista, ou seja, todos os recémnascidos filhos de mães que tiveram sífilis são considerados portadores da doença. Consequentemente, não há o cuidado em identificar os recém-nascidos realmente suscetíveis a apresentar sífilis congênita, sem distinção da forma de tratamento dessa condição no pré-natal, nem da fase da gestação em que houve a contaminação.

Por conseguinte, é importante que os profissionais que trabalham com programas de identificação precoce da deficiência auditiva infantil avaliem, junto à equipe médica, as condições do tratamento ao longo da gestação, bem como os testes sorológicos da mãe e de seu bebê assintomático à sífilis no momento da avaliação audiológica, para melhor interpretação dos resultados dos testes audiológicos ao longo do acompanhamento dessa população.

O atual estudo apresentou resultados inéditos com relação aos achados eletroacústicos e eletrofisiológicos, sugerindo que, nesses indivíduos, a cóclea se mostrou mais fragilizada diante dessa condição. Isto foi demonstrado pelo desempenho inferior na amplitude de resposta das EOAT, mesmo que de forma discreta. Já em relação à atividade neural, avaliada pelo PEATE-clique, não foram encontradas diferenças entre os grupos. Portanto, enfatiza-se que é necessário o acompanhamento do desenvolvimento auditivo, a fim de identificar, ou não, alterações audiológicas em longo prazo. Ressalta-se, também, a importância da realização de pesquisas com a mesma problemática, com uma maior casuística e dados contendo informação do followup auditivo desses indivíduos.

\section{CONCLUSÃO}

A amplitude de resposta das EOAT foi inferior, principalmente em $4,0 \mathrm{kHz}$, na orelha direita, para os recém-nascidos expostos à sífilis materna, adequadamente tratada na gestação. Por outro lado, as respostas eletrofisiológicas por meio do PEATE-clique foram semelhantes às encontradas na população de baixo risco para deficiência auditiva.

\section{REFERÊNCIAS}

1. Joint Committee on Infant Hearing. Year 2019. Position Statement: Principles and Guidelines for Early Hearing Detection and Intervention Programs. Pediatrics. 2019;4(2):1-44.

2. Becker GD. Late syphilitic hearing loss: a diagnostic and therapeutic dilemma. Laryngoscope. 1979;89(8):1273-88. http://dx.doi.org/10.1002/ lary.1979.89.8.1273. PMid:459660.

3. Sonda EC, Richter FF, Boschetti G, Casasola MP, Krumel CF, Machado CPH. Congenital Syphilis: literature review. Rev Epidemiol Control Infect. 2013;3(1):28-30. http://dx.doi.org/10.17058/reci.v3i1.3022.

4. Khetarpal S, Kempf E, Mostow E. Congenital syphilis: early- and late-stage findings of rhagades and dental anomalies. Pediatr Dermatol. 2011;28(4):401-3. http://dx.doi.org/10.1111/j.1525-1470.2010.01288.x. PMid:21133996.

5. Schmidt R, Carson PJ, Jansen RJ. Resurgence of Syphilis in the United States: an assessment of contributing factors. Infect Dis. 2019;12:1-9. http://dx.doi.org/10.1177/1178633719883282. PMid:31666795.

6. Brasil. Ministério da Saúde. Secretaria de Vigilância em Saúde. Boletim Epidemiológico Sífilis [Internet]. Brasília: Ministério da Saúde; 2019 [citado em 2020 Out 13]. Disponível em: http://www.aids.gov.br/pt-br/ pub/2019/boletim-epidemiologico-sifilis-2019

7. Pinilla G, Campos L, Durán A, Navarrete J, Muñoz L. Detección de Treponema pallidum subespecie pallidum para el diagnóstico de sífilis congénita mediante reacción en cadena de la polimerasa anidada. Biomedica. 2018;38(1):128-35. http://dx.doi.org/10.7705/biomedica. v38i0.3740. PMid:29676865.

8. Tsai S, Sun MY, Kuller JA, Rhee EHJ, Dotters-Katz S. Syphilis in pregnancy. Obstet Gynecol Surv. 2019;74(9):557-64. http://dx.doi. org/10.1097/OGX.0000000000000713. PMid:31830301.

9. Stanford KA, Hazra A, Schneider J. Routine opt-out syphilis screening in the emergency department: a public health imperative. Acad Emerg Med. 2020;27(5):437-8. http://dx.doi.org/10.1111/acem.13897. PMid:31802561.

10. Cavalcante PAM, Pereira RBL, Castro JGD, Cavalcante PAM, Pereira RBL, Castro JGD. Sífilis gestacional e congênita em Palmas, Tocantins, 2007-2014. Epidemiol Serv Saude. 2017 Mar;26(2):255-64. http:// dx.doi.org/10.5123/S1679-49742017000200003. PMid:28492767.

11. Brasil. Ministério da Saúde. Secretaria de Vigilância em Saúde. Departamento de DST, AIDS e hepatites virais. Boletim Epidemiológico Sífilis [Internet]. Brasília: Ministério da Saúde; 2020 [citado em 2020 Set 13]. Disponível em: http://www.aids.gov.br/pt-br/pub/2020/ boletim-sifilis-2020

12. Brasil. Ministério da Saúde. Secretaria de Vigilância em Saúde. Boletim Epidemiológico Sífilis [Internet]. Brasília: Ministério da Saúde; 2017 [citado em 2020 Ago 13]. Disponível em: http://www.aids.gov.br/ pt-br/pub/2017/boletim-epidemiologico-de-sifilis-2017

13. Gleich LL, Urbina M, Pincus RL. Asymptomatic congenital syphilis and auditory brainstem response. Int J Pediatr Otorhinolaryngol. 1994;30(1):11-3. http://dx.doi.org/10.1016/0165-5876(94)90046-9. PMid:8045690.

14. Chau J, Atashband S, Chang E, Westerberg BD, Kozak FK. A systematic review of pediatric sensorineural hearing loss in congenital syphilis. 
Int J Pediatr Otorhinolaryngol. 2009;73(6):787-92. http://dx.doi. org/10.1016/j.ijporl.2009.02.021. PMid:19321207.

15. Côrtes-Andrade IF, Bento DV, Lewis DR. Transient evoked otoacoustic emissions (TEOE): newborn hearing screening program protocols. Rev CEFAC. 2013;15(3):521-7. https://doi.org/10.1590/S151618462012005000062 .

16. Aidan D, Lestang P, And PA, Bonfils P. Characteristics of transientevoked otoacoustic emissions (TEOEs) in neonates. Acta Otolaryngol. 1997;117(1):25-30. http://dx.doi.org/10.3109/00016489709117986. PMid:9039476.

17. Silva DP, Martins RH. Analysis of transient otoacoustic emissions and brainstem evoked auditory potentials in neonates with hyperbilirubinemia. Braz J Otorhinolaryngol. 2009;75(3):381-6. http://dx.doi.org/10.1016/ S1808-8694(15)30656-X. PMid:19649489.
18. Santos AF, Durante AS, Almeida K, Taguchi CK, Greco MC. Características das emissões otoacústicas em lactentes expostos à medicação ototóxica. Rev Soc Bras Fonoaudiol. 2009;14(4):521-7. http://dx.doi.org/10.1590/S1516-80342009000400016.

19. Ribeiro GE, Silva DP, Montovani JC. Montovani. Transient evoked otoacoustic emissions and auditory brainstem response in infants with perinatal asphyxia. Int J Pediatr Otorhinolaryngol. 2016;89:136-9. http://dx.doi.org/10.1016/j.ijporl.2016.08.009. PMid:27619044.

20. Leite CA Fo, Lagreca LCC, Jesus NO, Corvaro CP, Ferrarini MAG, Monteiro AIMP, et al. Alterações auditivas em crianças expostas à toxoplasmose durante a gestação. Rev CEFAC. 2017 Jun;19(3):330-9. http://dx.doi.org/10.1590/1982-0216201719313516.

21. Padilha MAD, Maruta ECS, Azevedo MF. Ocorrência de alterações auditivas em lactentes expostos à transmissão vertical do HIV. Audiol Commun Res. 2018;23:e1965. http://dx.doi.org/10.1590/2317-64312017-1965. 